\title{
Natural fruit peels as teeth whitening agents on primary teeth an in vitro study
}

\author{
Research Article
}

\section{Archana P Betur ${ }^{*}$, Raju O S², Roopa K B ${ }^{3}$}

1. Lecturer, 2. Professor, 3. Professor, Department of Pedodontics and Preventive Dentistry, Bapuji Dental College and Hospital, Davanagere, Karnataka.

\begin{abstract}
Context: Dental Bleaching or tooth whitening represents a conservative cosmetic approach even for children and adolescents, for whom treatment may be readily accomplished without permanent alteration to the enamel surface thus limiting the need for lifelong maintenance. Aims: The aim of this study was to compare the efficacy of banana peel and orange peel as teeth whitening agents in deciduous dentition. Methods and Material: Twenty extracted primary anterior teeth were used in this study and each crown was sectioned mesiodistally to obtain 40 samples which were flattened to create a standardized enamel surface. After the staining procedure, 40 samples were allocated into 4 groups, i.e., 10 samples per group, the standard group (35\% Hydrogen peroxide), the control group (distilled water), banana peel and orange peel. The samples of each group were treated using the respective agents with standardized pressure and time. Results: The tooth shades were assessed using Digital Spectrophotometer at baseline, 1 week of staining, 1 week of bleaching and 1 week post bleaching. All the results were statistically analysed with Post Hoc Tukey's test. Both the study groups showed significant changes but, banana peel showed better efficacy than orange peel. Conclusions: Banana peel and orange peel can be considered for teeth whitening in deciduous dentition.
\end{abstract}

Key Words: Teeth whitening, Banana peel, Orange peel, Digital Spectrophotometer.

\section{Introduction}

Over the past few decades, tooth bleaching or whitening has become very popular in the field of aesthetic dentistry.(1) Since the 1800s, the dentists focused more on the in-office bleaching of the non-vital teeth discoloured as a result of trauma or endodontic treatment. By the late 1980s, the traditional bleaching shifted towards the home applied bleaching (tray bleaching) and other techniques for vital bleaching that could be applied both in the dental office and at home. (2)

Till date most of the bleaching materials are based primarily on either hydrogen peroxide or carbamide peroxide, although results vary depending on factors such as type of stain, age of patient, concentration of the active agent, and treatment time. However these peroxides have always been implicated in the concurrent damage of dental tissues leading to varying degrees of sensitivity during and after the procedure as well. Free radicals produced by them are known to be capable of reacting with proteins, lipids and nucleic acids, causing cellular damage. Clinical

\section{* Corresponding Author:}

\section{Archana P Betur}

Lecturer,

Department of Pedodontics and Preventive Dentistry,

Bapuji Dental College and Hospital,

Davanagere-04, Karnataka. India.

Email Id: archana.betur@gmail.com studies have also observed a higher prevalence of gingival irritation in patients using bleaching materials with higher concentrations.(4) Therefore the use of such whitening products in children and young adults is not just debatable but also lacks scientific evidence. Hence there is a need for bleaching agents which can effectively whiten the teeth without causing damage to the tender dental tissues of growing children.

Bananas are usually among the daily fruits preferred by a vast majority of children. The Banana fruit as well as the other parts of the plant have been used for their therapeutic benefit in traditional medicine. The high amounts of potassium, magnesium, and manganese in the banana peel could prove invaluable in removing stains and whitening teeth. In addition to the above minerals banana peel also contains high amounts of calcium and vitamin $\mathrm{D}$ which could possibly strengthen the mineral matrix concurrently.(4)

Similarly the white inner portion of an Orange peel, called as the Albedo, contains several compounds such as limonene, glucose, and soluble fiber pectin with potential stain removing abilities. In fact Limonene is commercially used in a variety of cleaning products and toothpastes. Unlike the fruit itself, the peel does not contain citric acid, and hence the potential risk of enamel decalcification is negated.(5)

Thus the aim of this study was to evaluate banana peel and orange peel as whitening agents in children since they do not have harmful abrasive effects like other whiteners and are inexpensive as well. 


\section{Subjects and Methods}

Preparation of the sample

Twenty freshly extracted deciduous anterior teeth without surface defects were used in this study. The roots present were cut with a diamond disc (flex, double sided - nti ${ }^{\circledR}$ diamond discs) and the pulp tissue was removed using spoon excavator. Then, the teeth were stored in $0.5 \%$ chloramine $-\mathrm{t}$ solution for one week. (3)

After disinfection, each crown was sectioned mesiodistally to obtain one enamel-dentin slab of $4 \times 4 \times 2$ $\mathrm{mm}$ dimension which was measured with a digital caliper(DC 500, Mitutoyo, Suzano, SP, Brazil) thus getting 40 samples and then the surface of each sample was flattened with diamond disc (Flex, Double Sided Nickel Titanium ${ }^{\circledR}$ Diamond Discs) for standardization. The samples were then washed and stored in distilled water at $37^{\circ} \mathrm{C}$ for 1 week until the starting of the experimental procedure.(3)

\section{Staining procedure}

In order to standardize the tooth shade, an artificial staining of the specimens was carried out. The prepared enamel surfaces were etched using 37\% phosphoric acid for 60 seconds and rinsed with water for 30 seconds. The specimens were then immersed for 10 min twice a day over a week in a standardized solution at room temperature in capped plastic containers. ${ }^{3}$ The standardized solution was renewed daily by mixing 15 gms of Boost(GlaxoSmithKline Consumer Healthcare Ltd, Patiala road, Nabha, Punjab, India) (consisting of malted barley, Sugar, wheat flour, milk solids, cocoa powder, glucose, salt, minerals, vitamins, protein isolate, natural colour, acidity regulator, nature identical flavouring substance) in 150 $\mathrm{ml}$ of distilled water thus making $5 \mathrm{ml}$ per sample. The specimens after each immersion period were washed and stored in distilled water at $37^{\circ} \mathrm{C}$.(3)

\section{Bleaching procedure}

The forty study samples were allocated into 4 equal groups, viz., standard group, control group, banana peel and orange peel. The samples of standard group, control group, Banana peel and Orange peel were treated using the respective agents twice a day for 5 min for 7 days. After which the specimens were washed and stored in distilled water at $37^{\circ} \mathrm{C}$ until the next treatment.(3)

\section{Method of Bleaching}

The samples of the standard group were treated for 5 min each with cotton dipped in 35\% Hydrogen peroxide. The control group were treated for $5 \mathrm{~min}$ each with cotton dipped in distilled water. A ripe banana was peeled from the bottom to avoid loose threads and the peel used to gently treat the samples for 5 min each.(6) Similarly an orange was peeled from the center, upside down without cutting through the flesh and the white of the peel used to gently treat the samples for 5 min each. (6)

\section{Tooth shade measurement}

The tooth shades were assessed using Digital Spectrophotometer at baseline, 1 week of staining, 1 week of bleaching and 1 week post bleaching. The digital Spectrophotometer (Konica Minolta Spectrophotometer CM-3301d, Marunouchi, Chiyoda, Tokyo, Japan) measures the tooth shades in the CIE L* $a^{*} b^{*}$ colour system, which was defined by the International Commission on Illumination. In the CIELAB colour system, the difference between the colour coordinates were calculated as $\Delta \mathrm{E}$.(3)

\section{Statistical analysis}

All the results were tabulated and appropriate statistical analysis was done using One way ANOVA followed by POST HOC Tukey's test. The level of confidence was established at $\alpha=0.05$.

\section{Results}

The tooth shade mean and standard deviation of the parameter $\left(\Delta \mathrm{E}^{*}\right)$ for the study groups at different evaluation periods are represented in table 1. At baseline all the four groups' readings were considered as zero. After staining all the four groups showed significant colour change from baseline $(\mathrm{p}<0.001)$. After bleaching, the banana group showed a statistically significant decrease in tooth shade than the orange group $(\mathrm{p}<0.001)$. The control group did not show significant decrease in tooth shade. The standard group showed most significant decrease in the tooth shade of all the other groups. Though the results of the standard group showed the highest decrease in the tooth shade, because of the side effects and contraindications of peroxides in deciduous dentition, it's effectiveness on deciduous dentition will not be taken into complete consideration while comparing the study groups. 1 week post bleaching results were not significantly different in any of the groups.

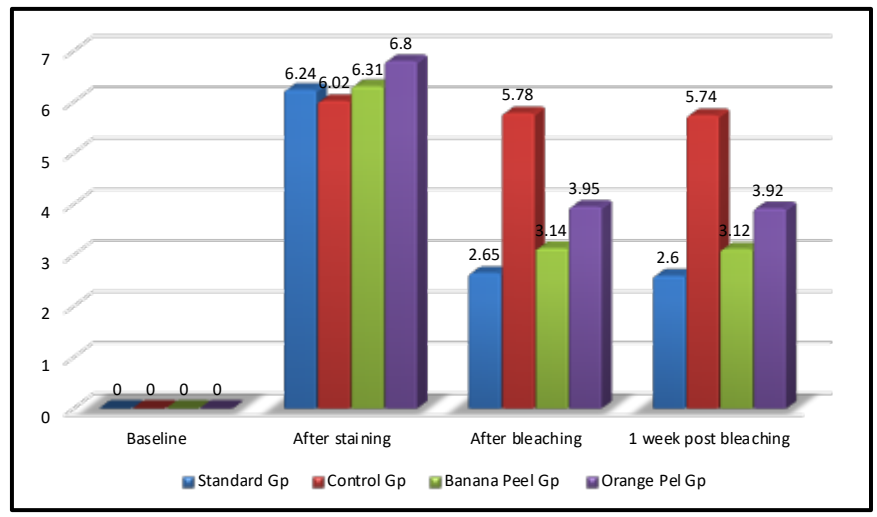

Graph 1: Mean of the groups; Standard Gp, Control Gp, Banana Peel Gp and Orange Peel Gp are plotted at baseline, after staining, after bleaching and 1 week post bleaching 
Table 1: Illustrating the results of one way ANOVA and Post HOC Tukey's test of the groups A - Control group, B - Banana peel and C - Orange peel at baseline, after staining, after bleaching and 1 week post bleaching (NS - Not Significant, * - Highly significant)

\begin{tabular}{|c|c|c|c|c|c|}
\hline & Groups & Baseline & After staining & After bleaching & 1 Week post bleaching \\
\hline \multirow{3}{*}{ Mean \pm S.D } & A & 0.0 & $6.03 \pm 1.04$ & $5.78 \pm 0.96$ & $5.74 \pm 0.94$ \\
& B & 0.0 & $6.31 \pm 1.09$ & $3.14 \pm 0.79$ & $3.12 \pm 0.78$ \\
\hline \multirow{2}{*}{ ANOVA } & C & 0.0 & $6.80 \pm 0.65$ & $4.95 \pm 0.74$ & $4.92 \pm 0.72$ \\
\cline { 2 - 6 } & F value & - & 1.71 & 26.08 & 27.01 \\
\hline \multirow{2}{*}{ Post HOC test } & P value & - & $0.20, \mathrm{NS}$ & $<0.001$ & $<0.001$ \\
& A - B & - & $0.78, \mathrm{NS}$ & $<0.001$ & $<0.001$ \\
\cline { 2 - 6 } & $\mathrm{A}-\mathrm{C}$ & - & $0.18, \mathrm{NS}$ & $0.009, \mathrm{NS}$ & $0.08, \mathrm{NS}$ \\
\hline
\end{tabular}

\section{Discussion}

Discolouration of the teeth is one of the most frequent reasons why a patient seeks dental care.(7) While it is more common in adults, significant tooth discolouration represents one possible indication for bleaching in children and adolescents as well. Bleaching represents a conservative first approach which changes the shade and appearance of teeth without using restorative materials.(8) However success of vital tooth bleaching as a non-invasive, conservative treatment for discoloured teeth has only gained acceptance in the recent past.(3) Although peroxides added with desensitizers have been deemed safe as tooth whiteners for adults, there are very limited studies supporting their use in children.(9)

Natural products have been used for several years in medical field. Over the last decade, a shift towards the usage of herbal medication in dentistry (for both prophylaxis and treatment) has turned out to be a safer and popular alternative more so with children.(10)

Banana has been traditionally used as a healing remedy for various kinds of diseases.(10) Scientific evidence attributes different phytochemicals isolated from the Banana fruit pulp, peel, seeds and flowers, as efficacious in treating diarrhoea, ulcerations, hypertension as well as teeth whitening.(4) Similarly Orange fruit is a commonly available source of vitamin $\mathrm{C}$ and dietary fiber. However its' white inner portion contains d-limonene which is used as a whitening agent in many toothpastes.(5)

Thus, in this study the peels of banana and orange have been used for teeth whitening in deciduous teeth by rubbing them onto the prepared enamel surfaces of respective groups for a standardized period of time. The results showed that both banana and orange peels were effective in removing the stains, but the banana peel proved to be a better whitening agent than the orange peel probably due to the composition and the action of the ingredients present viz ., potassium, magnesium and manganese which could prove invaluable in removing stains and whitening teeth as indicated by previous studies.(11) In fact potassium nitrate has been proven to have desensitizing effects and hence finds itself added to many whitening products. (12) Similarly magnesium chloride acts as an abrasive(13) and manganese gluconate tends to increase the bleaching efficacy.(14) The fact that orange peel also gave decent results could be due to the presence of d-limonene which is a proven stain remover.(5)

Considering the study groups evaluated in this study, it could be suggested that the use of peels of banana and orange could prove efficacious as well as a safe bleaching option for deciduous dentition. As this is an in vitro study carried out for a short duration, further long term studies and 'in - vivo' clinical trials are needed to determine the long term stability of teeth whitening procedures performed using banana and orange peels.

\section{Conclusion}

Within the limitations of this study, it may be concluded that banana and orange peels could be used as potential whitening agents on deciduous teeth.

\section{References}

1. Haywood VB, Heymann HO. Nightguard vital bleaching.Quintessence Int 1989;20:173-176.

2. Cohen S, Parkins FM. Bleaching tetracyclinestained vital teeth. Oral Surg 1970;29:465-471.

3. Sonia Saeger Meireles, Silvia Terra Fontes, Luiz Antonio Afonso Coimbra,Alvaro Della Bona, Flavio Fernando Demarco. Effectiveness of different carbamide peroxide concentrations used for tooth bleaching: an in vitro study. Journal of Applied Oral Science April/May 2012;20(2)

4. Mohammad Zafar Imam and Saleha Akter. Musa paradisiaca L. and Musa sapientum L.: A Phytochemical and Pharmacological Review. Journal of Applied Pharmaceutical Science 2011;01 (05): 14-20.

5. Xie P, Lu J, Wan H, Hao Y. Effect of toothpaste containing d-limonene on natural extrinsic smoking stain: a 4-week clinical trial. Am J Dent 2010 Aug;23(4):196-200.

6. How to whiten teeth with Banana peel and Orange peel (http://www.ehow.com/how 4807031 whitenteeth-banana-peel-orange-peel.html) accessed on $20^{\text {th }}$ May 2013.

7. Watts A, Addy M. Tooth discolouration and staining: a review of the literature. $\mathrm{Br}$ Dent $\mathrm{J}$ 2001;190:309-316.

8. Kelvin J Donly, Adriana Segura Donly, Laila Baharloo, Edith Rojas Candelas. Tooth whitening in Children.Compendium / Special issue 2002;23(1A):22-28. 
9. Sean S Lee, Wu Zhang, D. Harvey. Tooth whitening in Children and Adolescents : A literature review. Pediatr Dent 2005;27:362-368.

10. Ola Moustafa Omar et al. Alternative Medicine: Implications on Dentistry Alternative and Integrative Medicine, Omar, Altern Integ Med 2013, 1:1.

11. Chemical composition of Musa sapientum (Banana) peels. Journal of Food technology 2008;6(6): 263-266.
12. Tam L et al Effect of potassium nitrate and fluoride on carbamide peroxide bleaching. Quintessence Int. 2001 Nov-Dec;32(10):766-70.

13. Hooper $\mathrm{S}$ et al, Investigation of erosion and abrasion on enamel and dentine: a model in situ using toothpastes of different abrasivity. J Clin Periodontol 2003 Sep;30(9):802-8.

14. Batista GR et al, The influence of chemical activation on tooth bleaching using $10 \%$ carbamide peroxide. Oper Dent. 2011 Mar-Apr;36(2):162-8. 